\title{
Remedial Action
}

National Cancer Institute

\section{Source}

National Cancer Institute. Remedial Action. NCI Thesaurus. Code C53605.

An action taken in order to cure or restore to health. 